\title{
Selecting microscopic Equations of State
}

\author{
G. Taranto ${ }^{1}$, M. Baldo ${ }^{1}$, and G. F. Burgio ${ }^{1}$ \\ ${ }^{1}$ INFN Sezione di Catania, and Dipartimento di Fisica e Astronomia, \\ Universitá di Catania, Via Santa Sofia 64, 95123 Catania, Italy
}

\begin{abstract}
We compare a set of equations of state derived within microscopic many-body approaches, and study their predictions as far as phenomenological data on nuclei from heavy ion collisions, and astrophysical observations on neutron stars are concerned. We find that all the data, taken together, put strong constraints not easy to be fulfilled accurately. However, no major discrepancies are found among the selected equations of state and with respect to the data. The results provide an estimate of the uncertainty on the theoretical prediction at a microscopic level of the nuclear equation of state.
\end{abstract}

PACS numbers: $26.60 . \mathrm{Kp}, 26.60 .-\mathrm{c}, 21.65 .+\mathrm{f}, 24.10 . \mathrm{Cn}$, 97.60.Jd 21.65.Mn.

\section{INTRODUCTION}

A convergent effort of experimental and theoretical nuclear physics has been developing along several years to determine or to constrain the Equation of State (EoS) of nuclear matter. Two main areas of research have provided relevant hints in this direction. The experimental data on heavy ion collisions have been systematically analyzed on the basis of detailed simulations, in order to constrain not only the density dependence of the EoS, but also its isospin dependence. Excellent reviews on this subject can be found in the literature [1, 2]. On the other hand, the analysis of astrophysical observational data, noticeably on compact objects, are of great relevance for the study of the nuclear EoS. The results in this field of research can be considered complementary to the ones that can be obtained within the heavy ion research activity, because nuclear matter is involved in different physical conditions. In Neutron Stars (NS) nuclear matter is present in beta equilibrium from very low density to several times saturation density, and it is therefore extremely asymmetric, much more than nuclei in laboratory. Despite the different physical situations, an accurate microscopic theory of nuclear matter is expected to be able to explain correctly the data obtained in both physical realms. For a pedagogical introduction to the field see ref. [3]. Another more phenomenological approach is based on the Energy Density Fuctional (EDF) method, in particular the Skyrme forces scheme. In this approach a phenomenological force or EDF includes a certain set of parameters that are fixed by fitting the binding energy of nuclei throughout the mass table. Some of these forces are adjusted also to microscopic nuclear matter EoS. Recently, an ample set of Skyrme forces, that have been presented in the literature, has been analyzed and confronted with the available constraints on the nuclear EoS obtained from heavy ion reactions and astrophysical objects [4]. The few Skyrme forces that passed these tests have been then used to predict the binding energy of a wide set of nuclei [5], and none seems to perform satisfactorily well in this case. These results show clearly that it is not at all trivial to satisfy the constraints coming from astrophysical and heavy ion data, as it was already found in ref.[6]. Furthermore, if the nuclear mass data are included, it seems very difficult to reproduce all the data sets. It looks that the phenomenological forces are not flexible enough to this purpose. In this paper we follow a complementary approach. We focus on the microscopic theory of nuclear matter and the corresponding EoS's that have been developed so far. Adopting the same line as in ref.[4], we test the predictions of different microscopic manybody approaches with respect to the constraints coming from experiments and phenomenology. The aim of this analysis is to establish to what extent the data can be reproduced within a microscopic approach and the accuracy of the present theory of nuclear matter.

This paper is organized as follows. In Sect.II a review of the currently used microscopic many-body methods is presented, whereas results are discussed in Sect. III. Finally, in Sect. IV we draw our conclusions.

\section{EOS OF NUCLEAR MATTER IN MICROSCOPIC APPROACHES}

Empirical properties of infinite nuclear matter can be calculated using many different theoretical approaches. In this paper we concentrate on the microscopic ones, the only input being a realistic free nucleon-nucleon $(\mathrm{NN})$ interaction with parameters fitted to $\mathrm{NN}$ scattering phase shifts in different partial wave channels, and to properties of the deuteron. In the following we discuss the non-relativistic BruecknerHartree-Fock (BHF) method [7] and its relativistic counterpart, the Dirac-Bruckner- Hartree-Fock (DBHF) approximation [8], and the Variational method [9]. A relatively more recent approach is based on the Chiral effective Field Theory, which has been extensively applied to Nuclear Matter. For a review see ref. [10, 11]. It establishes a link with the underlying QCD structure of strong interaction, and it has reached a high degree of sophistication [12-16]. We will not discuss this approach, because the corresponding EoS is based on a low momentum expansion, and therefore its behaviour at high density is uncertain. Hence, a comparison with the other microscopic EoS's would be incomplete. However, the EoS from the Chiral approach turns out to be close to the variational EoS [10], and therefore the parameters near saturation are expected to be quite similar. 


\section{A. The Brueckner-Bethe-Goldstone approach}

The Brueckner-Bethe-Goldstone (BBG) theory is based on a linked cluster expansion of the energy per nucleon of nuclear matter (see Ref. [7], chapter 1 and references therein). The basic ingredient in this many-body approach is the Brueckner reaction matrix $G$, which is the solution of the BetheGoldstone equation

$$
G[\rho ; \omega]=v+\sum_{k_{a} k_{b}} v \frac{\left|k_{a} k_{b}\right\rangle Q\left\langle k_{a} k_{b}\right|}{\omega-e\left(k_{a}\right)-e\left(k_{b}\right)} G[\rho ; \omega],
$$

where $v$ is the bare $\mathrm{NN}$ interaction, $\rho$ is the nucleon number density, and $\omega$ the starting energy. The single-particle energy $e(k)$ (assuming $\hbar=1$ here and throughout the paper),

$$
e(k)=e(k ; \rho)=\frac{k^{2}}{2 m}+U(k ; \rho),
$$

and the Pauli operator $Q$ determine the propagation of intermediate baryon pairs. The Brueckner-Hartree-Fock (BHF) approximation for the single-particle potential $U(k ; \rho)$ using the continuous choice is

$$
U(k ; \rho)=\operatorname{Re} \sum_{k^{\prime} \leq k_{F}}\left\langle k k^{\prime}\left|G\left[\rho ; e(k)+e\left(k^{\prime}\right)\right]\right| k k^{\prime}\right\rangle_{a},
$$

where the subscript " $a$ " indicates antisymmetrization of the matrix element. Due to the occurrence of $U(k)$ in Eq. (2), they constitute a coupled system that has to be solved in a self-consistent manner for several momenta of the particles involved, at the considered densities. In the BHF approximation the energy per nucleon is

$$
\frac{E}{A}=\frac{3}{5} \frac{k_{F}^{2}}{2 m}+\frac{1}{2 \rho} \sum_{k, k^{\prime} \leq k_{F}}\left\langle k k^{\prime}\left|G\left[\rho ; e(k)+e\left(k^{\prime}\right)\right]\right| k k^{\prime}\right\rangle_{a} .
$$

In this scheme, the only input quantity we need is the bare NN interaction $v$ in the Bethe-Goldstone equation (1). The nuclear EoS can be calculated with good accuracy in the Brueckner two hole-line approximation with the continuous choice for the single-particle potential, since the results in this scheme are quite close to the calculations which include also the three hole-line contribution [17].

However, it is commonly known that non-relativistic calculations, based on purely two-body interactions, fail to reproduce the correct saturation point of symmetric nuclear matter. One of the well known results of several studies, that lasted for about half a century, is the need of introducing three-body forces (TBFs). In our approach the TBF is reduced to a density dependent two-body force by averaging over the position of the third particle, assuming that the probability of having two particles at a given distance is reduced according to the two-body correlation function [18].

In this work we will illustrate results for two different approaches to the TBF's, i.e. a phenomenological and a microscopic one. The phenomenological approach is based on the so-called Urbana model, which consists of an attractive term due to two-pion exchange with excitation of an intermediate $\Delta$ resonance, and a repulsive phenomenological central term [19]. We introduced the same Urbana three-nucleon model within the BHF approach. Those TBF's produce a shift of about $+1 \mathrm{MeV}$ in energy and $-0.01 \mathrm{fm}^{-3}$ in density. This adjustment is obtained by tuning the two parameters contained in the TBF's, and was performed to get an optimal saturation point (the minimum) (for details see Ref. [18]).

The connection between two-body and three-body forces within the meson-nucleon theory of nuclear interaction is extensively discussed and developed in references [20, 21]. At present the theoretical status of microscopically derived TBF's is still quite rudimentary, however a tentative approach has been proposed using the same meson-exchange parameters as the underlying NN potential. Results have been obtained with the Argonne $v_{18}$, the Bonn B, and the Nijmegen 93 potentials [20].

In the past years, the BHF approach has been extended in order to include the hyperon degrees of freedom [22], which play an important role in the study of neutron star matter. However, in this paper we are mainly interested on the properties of the nucleonic EoS, therefore this issue will not be discussed further.

\section{B. The Relativistic approach}

The relativistic framework is the one on which the nuclear EoS should be ultimately based. The best relativistic treatment developed so far is the Dirac-Brueckner (DBHF) approach [8]. The DBHF method can be developed in analogy with the non-relativistic case, i.e. the nucleon inside the nuclear medium is viewed as a dressed particle in consequence of its two-body interaction with the surrounding nucleons. The two-body correlations are described by introducing the in-medium relativistic $G$-matrix. The DBHF scheme can be formulated as a self-consistent problem between the single particle self-energy $\Sigma$ and the $G$-matrix. Schematically, the equations can be written

$$
\begin{aligned}
G & =V+i \int V Q g g G \\
\Sigma & =-i \int_{F}(\operatorname{Tr}[g G]-g G)
\end{aligned}
$$

where $Q$ is the Pauli operator which projects the intermediate two particle momenta outside the Fermi sphere, as in the BBG G-matrix equation (1), and $g$ is the single particle Green's function, which fulfills the Dyson equation

$$
g=g_{0}+g_{0} \Sigma g
$$

where $g_{0}$ is the (relativistic) single particle Green's function for a free gas of nucleons, and $\Sigma$ is the nucleon self-energy which expresses the influence of the surrounding nucleons. The self-energy can be expanded in the covariant form

$$
\Sigma\left(k, k_{F}\right)=\Sigma_{s}\left(k, k_{F}\right)-\gamma_{0} \Sigma_{0}\left(k, k_{F}\right)+\gamma \cdot \mathbf{k} \Sigma_{v}
$$

where $\gamma_{\mu}$ are the Dirac gamma matrices, and the coefficients of the expansion are scalar functions, which in general depend 
on the modulus $|\mathbf{k}|$ of the three-momentum and on the energy $k_{0}$. The free single particle eigenstates, which determine the spectral representation of the free Green's function, are solutions of the Dirac equation

$$
\left[\gamma_{\mu} k^{\mu}-M\right] u(k)=0
$$

where $u$ is the Dirac spinor at four-momentum $k$. For the full single particle Green's function $g$ the corresponding eigenstates satisfy

$$
\left[\gamma_{\mu} k^{\mu}-M+\Sigma\right] u(k)^{*}=0
$$

Inserting the above general expression for $\Sigma$, eq. 8 , after a little manipulation one gets

$$
\left[\gamma_{\mu} k^{\mu *}-M^{*}\right] u(k)^{*}=0
$$

with

$$
k^{0^{*}}=\frac{k^{0}+\Sigma_{0}}{1+\Sigma_{v}} ; k^{i^{*}}=k^{i} \quad ; \quad M^{*}=\frac{M+\Sigma_{s}}{1+\Sigma_{v}}
$$

This is the Dirac equation for a single particle in the medium, and the corresponding solution is the spinor

$$
u^{*}(\mathbf{k}, s)=\sqrt{\frac{E_{\mathbf{k}}^{*}+M^{*}}{2 M^{*}}}\left(\begin{array}{c}
1 \\
\frac{\sigma \cdot k}{E_{\mathbf{k}}^{*}+M^{*}}
\end{array}\right) \chi_{s} ; E_{\mathbf{k}}^{*}=\sqrt{\mathbf{k}^{2}+M^{* 2}} .
$$

In line with the Brueckner scheme, within the BBG expansion, in the self-energy of equation (6) only the contribution of the single particle Green's function pole is considered. Furthermore, negative energy states are neglected and one gets the usual self-consistent condition between self-energy and scattering $G$-matrix.

In any case, the medium effect on the spinor of equation (13) is to replace the vacuum value of the nucleon mass and three-momentum with the in-medium values of equation (12). This means that the in-medium Dirac spinor is "rotated" with respect to the corresponding one in vacuum, and a positive (particle) energy state in the medium has some nonzero component on the negative (anti-particle) energy state in vacuum. In terms of vacuum single nucleon states, the nuclear medium produces automatically anti-nucleon states which contribute to the self-energy and to the total energy of the system. It has been shown in ref. [23] that this relativistic effect is equivalent to the introduction of well defined TBF's at the non-relativistic level. These TBF's turn out to be repulsive, and consequently produce a saturating effect. Actually, including in BHF only these particular TBF's, one gets results close to DBHF calculations, see ref.[24]. Generally speaking, the DBHF gives in general a better saturation point than BHF, and the corresponding EoS turns out to be stiffer above saturation than the one calculated from the $\mathrm{BHF}+\mathrm{TBF}$ method.

In the relativistic context the only NN potentials which have been developed are the ones of one boson exchange (OBE) type. In the calculations shown here the Bonn A potential is used [25].

\section{The Variational method}

In the variational method [9] one assumes that the ground state wave function $\Psi$ can be written in the form

$$
\Psi\left(r_{1}, r_{2}, \ldots \ldots\right)=\Pi_{i<j} f\left(r_{i j}\right) \Phi\left(r_{1}, r_{2}, \ldots .\right),
$$

where $\Phi$ is the unperturbed ground state wave function, properly antisymmetrized, and the product runs over all possible distinct pairs of particles. The correlation factor is here determined by the variational principle, i.e. by imposing that the mean value of the Hamiltonian gets a minimum

$$
\frac{\delta}{\delta f} \frac{\langle\Psi|H| \Psi\rangle}{\langle\Psi \mid \Psi\rangle}=0
$$

In principle this is a functional equation for the correlation function $f$, which however can be written explicitly in a closed form only if additional suitable approximations are introduced. The function $f\left(r_{i j}\right)$ is assumed to converge to 1 at large distance and to go rapidly to zero as $r_{i j} \rightarrow 0$, to take into account the repulsive hard core of the NN interaction. Furthermore, at distance just above the core radius a possible increase of the correlation function beyond the value 1 is possible.

For nuclear matter it is necessary to introduce a channel dependent correlation factor, which is equivalent to assume that $f$ is actually a two-body operator $\hat{F}_{i j}$. One then assumes that $\hat{F}$ can be expanded in the same spin-isospin, spin-orbit and tensor operators appearing in the NN interaction. Momentum dependent operators, like spin-orbit, are usually treated separately. The product in equation (14) must be then symmetrized since the different terms do not commute anymore.

If the two-body NN interaction is local and central, its mean value is directly related to the pair distribution function $g(\mathbf{r})$

$$
<V>=\frac{1}{2} \rho \int d^{3} r v(r) g(\mathbf{r}),
$$

where

$$
g\left(\mathbf{r}_{\mathbf{1}}-\mathbf{r}_{\mathbf{2}}\right)=\frac{\int \Pi_{i>2} d^{3} r_{i}\left|\Psi\left(r_{1}, r_{2} \ldots\right)\right|^{2}}{\int \Pi_{i} d^{3} r_{i}\left|\Psi\left(r_{1}, r_{2} \ldots\right)\right|^{2}}
$$

The main job in the variational method is to relate the pair distribution function to the correlation factors $F$. Again, in nuclear matter also the pair distribution function must be considered channel dependent and the relation with the correlation factor becomes more complex. In general this relation cannot be worked out exactly, and one has to rely on some suitable expansion. Furthermore, three-body or higher correlation functions must in general be introduced, which will depend on three or more particle coordinates and describe higher order correlations in the medium. Many excellent review papers exist in the literature on the variational method and its extensive use for the determination of nuclear matter $\operatorname{EoS}[9,26]$. The best known and most used variational nuclear matter EoS is the one calculated by Akmal, Pandharipande and Ravenhall [27]. In their paper the authors showed calculations using the Argonne $v_{18} \mathrm{NN}$ interaction [28], with boost corrections to 
the two-nucleon interaction, which give the leading relativistic effect of order $(v / c)^{2}$, as well as three-nucleon interactions modeled with the Urbana force explained above. This EoS will be tested in the present paper.

\section{RESULTS AND DISCUSSION}

\section{A. Phenomenology of nuclei}

One of the main goals of the microscopic many-body methods is the correct reproduction of the nuclear matter saturation point. The non-relativistic approaches typically lead to an over prediction of the saturation density $\rho_{0}=0.17 \pm$ $0.03 \mathrm{fm}^{-3}$ of symmetric nuclear matter (SNM), at which the binding energy $E / A$ per nucleon reaches its minimum. The empirical value $E / A\left(\rho_{0}\right) \approx-16 \mathrm{MeV}$ can be extracted from the semi-empirical mass formula or from the extrapolation of binding energies of heavy nuclei. In Table I we display theoretical calculations of saturation properties of SNM for the different approaches illustrated above.

In the case of the BHF scheme with the Urbana three-body forces (first line of Table I), the reported values for the saturation point have been obtained by a polynomial fit to the numerically calculated EoS. The fit was performed with the constraint to reproduce the empirical saturation density of $0.16 \mathrm{fm}^{-3}$ while the corresponding energy was tuned to give the optimal value within a recent Energy Density Functional (EDF) approach based on this BHF EoS [29]. The shifts in the values for the density and energy at saturation from the original numerical values $\left(0.17 \mathrm{fm}^{-3}\right.$ and $\left.-15.2 \mathrm{MeV}\right)$ have only marginal effects on the other physical parameters reported in Table I. For the BHF case with microscopic three-body forces (second and third lines), no application to an EDF scheme was performed, so the reported saturation point corresponds to the numerical calculations.

In the case of the considered variational method (fourth line), in the quoted reference the original numerical EoS was corrected around saturation with an extra binding energy in functional form in order to reproduce the empirical saturation point, as reported in Table I. This correction is large as much as $4 \mathrm{MeV}$. We do not know how much this correction could affect the values of the other physical parameters reported in the table, but we used the corrected EoS, since it is the one recommended in the original paper [27] and widely used in the literature. In particular it was used to construct the functional NRAPR [30] and in many astrophysical studies on Neutron Stars, see e.g. ref. [31]. Finally, in the DBHF approach there is no need to introduce three-body forces, although the saturation point is slightly shifted to higher density. We therefore report the calculated values without fine tuning or corrections.

It has to be stressed that in all cases the EoS above saturation was in no way corrected.

In the third column of the same table we show the values predicted for the incompressibility at the saturation point. For practical reasons, it is customary among nuclear physicists to use the definition

$$
K_{0}=k_{F}^{2}\left(\frac{d^{2} E / A}{d k_{F}^{2}}\right)_{\rho_{0}}
$$

which has the dimension of an energy. The values of the incompressibility extracted from the EoS in the many-body methods are displayed in Table \. The values are typical of a pretty soft EoS at saturation, in agreement with the values extracted from the phenomenology of monopole oscillations [32]. One finds indeed that a correlation exists between incompressibility and position of the monopole Giant Resonance, so that, in principle it is possible to extract from the experimental data the value of the incompressibility in nuclear matter. At present, the constraints on the value of the nuclear matter incompressibility from the monopole excitation are not so tight. It can be approximately constrained between 210 and $250 \mathrm{MeV}$ [4], though a more refined value can be expected to come out in the near future from additional analysis of phenomenological data.

These values are compatible with the ones obtained from the experiments on sub-threshold kaon production, noticeably the ones from the KaoS [33] and FOPI [34] collaborations. The optimal energy for this type of investigation is close or even below two-body threshold, since then the only way to produce the kaons is by compression of the matter. Since at threshold the production rate increases steeply, there is a strong sensitivity to the value of the maximum density reached during the collision, and this is an ideal situation for studying the EoS and its incompressibility. The comparison of the simulations with the experimental data on $K^{+}$production points in the direction of a soft EoS. However it has to be kept in mind that, in the simulations, kaon production occurs at density $\rho \geq 2-3 \rho_{0}$, and therefore this set cannot be directly compared to the one of the monopole oscillations. In any case, a stiff EoS above saturation seems to be excluded from this analysis, as it is apparent in ref. [35].

Further important properties characterizing the nuclear matter are the symmetry energy at saturation $S\left(\rho_{0}\right)$ and its density derivative $L$, which are displayed in columns 4 and 5 of Table \. For both those quantities experimental data do exist, and will be discussed in the next subsections. The usual way of calculating them is to assume asymmetric nuclear matter, where the proton number $N_{p}$ is different from the neutron number $N_{n}$, with $N=N_{n}+N_{p}$. In this case, the EoS of nuclear matter has to be generalized. Defining

$$
\beta=\frac{N_{n}-N_{p}}{N_{n}+N_{p}}=\frac{\rho_{n}-\rho_{p}}{\rho}
$$

as the "asymmetry" parameter, one easily gets

$$
\begin{aligned}
\frac{E}{A}(\rho, \beta) & =\frac{E}{A}(\rho, 0)+E_{\text {sym }}(\rho) \beta^{2} \\
& =\frac{E}{A}\left(\rho_{0}\right)+\frac{1}{18} K_{0} e^{2}+\left[S_{0}-\frac{1}{3} L e+\frac{1}{8} K_{\text {sym }} e^{2}\right] \beta^{2}
\end{aligned}
$$

where $e=\left(\rho-\rho_{0}\right) / \rho_{0}, K_{0}$ is the incompressibility at the saturation point, $S_{0}=S\left(\rho_{0}\right)$ is the symmetry energy coefficient at 


\begin{tabular}{|l|c|c|c|c|c|}
\hline EoS & $\rho_{0}\left(\mathrm{fm}^{-3}\right)$ & $\frac{E}{A}(\mathrm{MeV})$ & $K_{0}(\mathrm{MeV})$ & $S_{0}(\mathrm{MeV})$ & $L(\mathrm{MeV})$ \\
\hline \hline$B H F, A v_{18}+U V I X$ & 0.16 & -15.98 & 212.4 & 31.9 & 52.9 \\
\hline$B H F, A v_{18}+$ micro $T B F$ & 0.2 & -15.5 & 236. & 31.3 & 82.7 \\
\hline$B H F$, Bonn B+micro $T B F$ & 0.17 & -16. & 254. & 30.3 & 59.2 \\
\hline$A P R, A v_{18}+U V I X$ & 0.16 & -16. & 247.3 & 33.9 & 53.8 \\
\hline DBHF, Bonn $A$ & 0.18 & -16.15 & 230. & 34.4 & 69.4 \\
\hline
\end{tabular}

TABLE I: Calculated properties of symmetric nuclear matter.

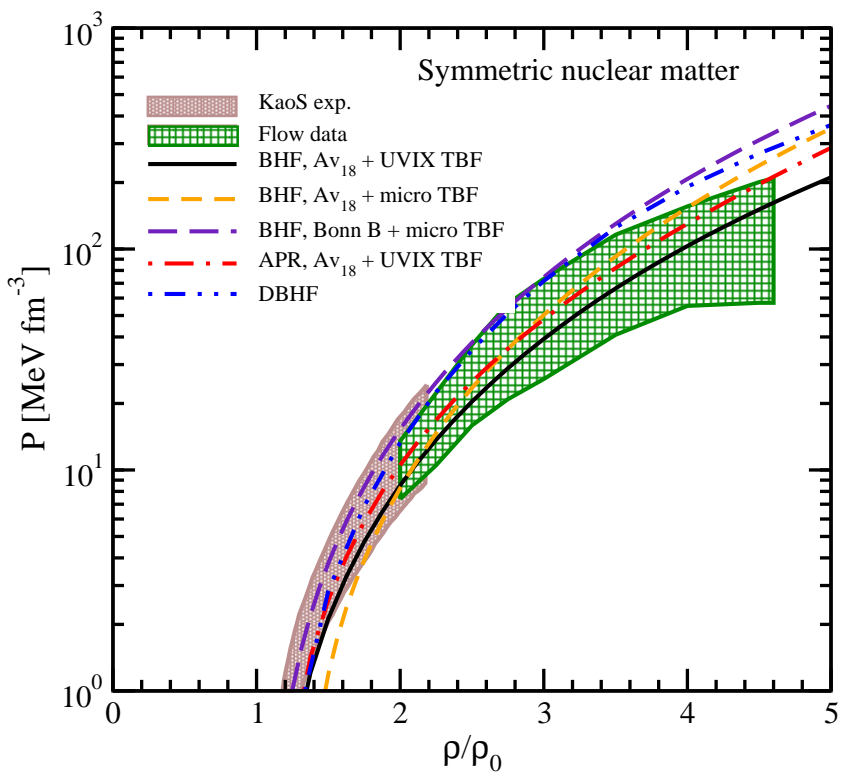

FIG. 1: (Color online) Pressure as a function of baryon density for symmetric nuclear matter. See text for details.

saturation and the parameters $L$ and $K_{\text {sym }}$ in the square bracket characterize the density dependence of the symmetry energy around the saturation point. The values in Table I of these physical parameters are obtained, for each one of the indicated microscopic many-body EoS, from their analytical form or by a polynomial fit to the numerical values reported in the original papers.

\section{B. Heavy ions phenomenology}

In the last two decades intensive studies of heavy ion reactions at energies ranging from few tens to several hundreds $\mathrm{MeV}$ per nucleon (hereafter indicated as MeV/A) have been performed. The main goal has been the extraction from the data of the gross properties of the nuclear EoS. It can be expected that in heavy ion collisions at large enough energy nuclear matter is compressed and that, at the same time, the two partners of the collisions produce flows of matter. In principle the dynamics of the collisions should be connected with the properties of the nuclear medium EoS and its viscosity. In the so called "multifragmentation" regime, after the collision numerous nucleons and fragments of different sizes are emitted, and the transverse flow, which is strongly affected by the

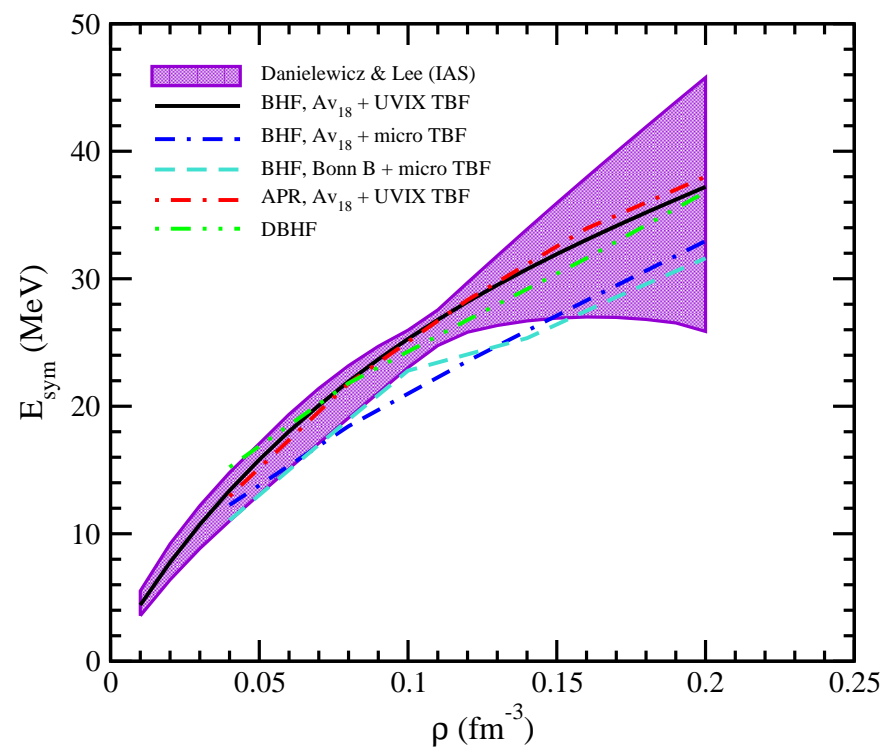

FIG. 2: (Color online) The symmetry energy is displayed as a function of the nucleon density. The purple zone represents the recent data by P. Danielewicz, whereas the different curves are the results of the microscopic many-body methods.

matter compression during the collision, can be measured.

Based on numerical simulations, in reference [36] a phenomenological range of densities was proposed where any reasonable EoS for symmetric nuclear matter should pass through in the pressure vs. density plane. The plot is reproduced in Fig 1, where a comparison with the microscopic calculations discussed in Section $\Pi$ is made. The green dashed box represents the results of the numerical simulations of the experimental data discussed in ref.[36], and the brown filled region represents the experimental data on kaons production [35]. We notice that the EoS calculated with the BHF and the variational methods including UVIX three-body forces look in agreement with the data in the full density range. On the other hand, the BHF EoS obtained using microscopic TBF's are only marginally compatible with the experimental data, as well as the DBHF EoS, showing that they are too repulsive already at density $\rho \geq 3 \rho_{0}$ if the Bonn potentials are used. Though, it has to be stressed that all EoS are compatible with the data around the saturation density, i.e. their incompressibility is as soft as required by the data. However, the values of the incompressibility do not characterize completely the EoS, since it is density dependent, but in any case the analysis indi- 
cates that the EoS al low density must be soft.

A further constraint on the EoS is given by the symmetry energy, which has been extensively studied both from the theoretical and experimental point of view in ref.[37]. The symmetry energy is displayed in Fig 2 as a function of the nucleon density. The purple region is the result of a recent analysis performed by P. Danielewicz on the isobaric analog states (IAS) in nuclei [38]. This stems from the charge independence of nuclear interactions, i.e. strong interactions between nucleons in the same state do not depend on whether the nucleons are protons or neutrons. Therefore the energy difference between the ground state of a nucleus with $N>Z$ and the isobaric analogs of the ground states of neighboring isobars are given by the symmetry energy, and the Coulomb contributions to the binding energy can be determined using the IAS. Many such states have been identified, and by fitting the available data on the IAS, Danielewicz and Lee obtained the constraint shown as a purple region in Fig 2 We observe that all EoS give results in very good agreement with the experimental data, except the ones of BHF with microscopic TBF at densities below the saturation density.

In Fig 3 we display the slope $L$ as a function of the symmetry energy at saturation density, which has been widely discussed in ref.[37]. Several experimental data are displayed. The blue band represents experimental data from HIC, obtained from the neutron and proton spectra from central collisions for ${ }^{124} \mathrm{Sn}+{ }^{124} \mathrm{Sn}$ and ${ }^{112} \mathrm{Sn}+{ }^{112} \mathrm{Sn}$ reactions at 50 $\mathrm{MeV} / \mathrm{A}$ [39]. At the same incident energy, isospin diffusion was investigated. We remind that isospin diffusion in HIC depends on the different $N / Z$ asymmetry of the involved projectiles and targets, hence it is used to probe the symmetry energy [2, 40, 41]. The full red circle shows the results from isospin diffusion observables measured for collisions at a lower beam energy of $35 \mathrm{MeV}$ per nucleon [42].

Transverse collective flows of hydrogen and helium isotopes as well as intermediate mass fragments with $Z<9$ have also been measured at incident energy of $35 \mathrm{MeV} / \mathrm{A}$ in ${ }^{70} \mathrm{Zn}+{ }^{70} \mathrm{Zn},{ }^{64} \mathrm{Zn}+{ }^{64} \mathrm{Zn},{ }^{64} \mathrm{Ni}+{ }^{64} \mathrm{Ni}$ reactions and compared to transport calculations. The analysis yielded values denoted by the full squares [43].

The box labelled by FRDM (finite-range droplet model) represents a refinement of the droplet model [44], and includes microscopic "shell" effects and the extra binding associated with $N=Z$ nuclei. The FRDM reproduces nuclear binding energies of known nuclei within $0.1 \%$, and allows determination of both $S_{0}=32.5 \pm 0.5 \mathrm{MeV}$ and $L=70 \pm 15 \mathrm{MeV}$.

In Fig 3 the other boxes represent experimental data obtained from measurements of the neutron skin thickness. In light nuclei with $N \approx Z$, the neutrons and protons have similar density distributions. With increasing the neutron number $N$, the radius of the neutron density distribution becomes larger than that of the protons, reflecting the pressure of the symmetry energy. The measurement of the neutron skin thickness is made on the stable nucleus ${ }^{208} \mathrm{~Pb}$, which has a closed neutron shell with $N=126$ and a closed proton shell with $Z=82$, hence it is very asymmetric and the neutron skin is very thick. The possibility of measurements of the neutron radius in ${ }^{208} \mathrm{~Pb}$ by the experiment PREX at Jefferson Labora-

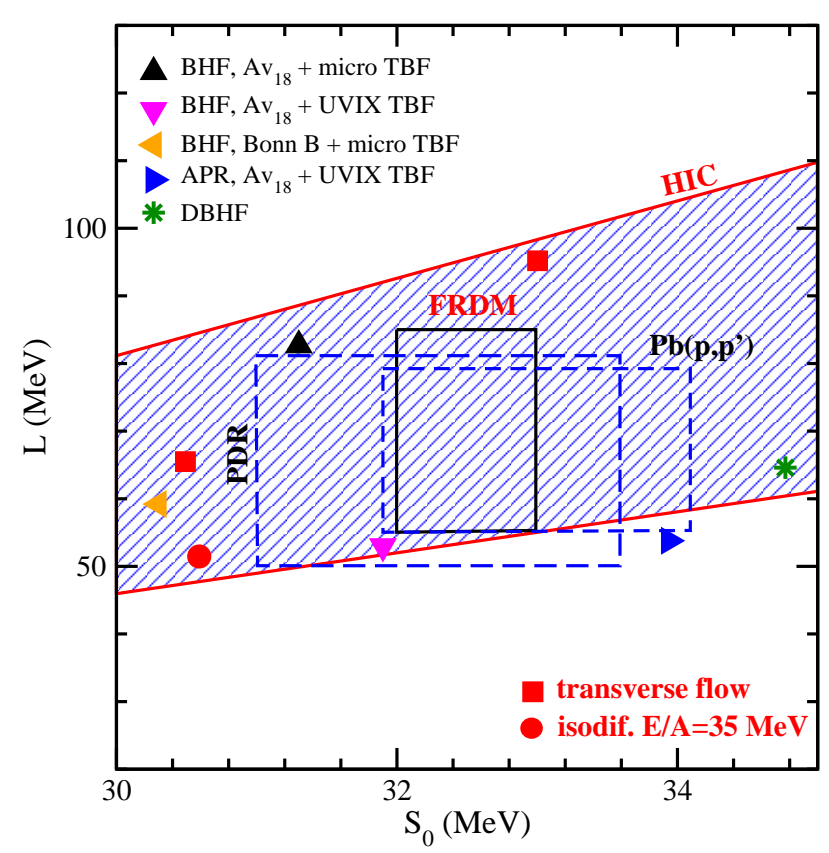

FIG. 3: (Color online) The derivative of the symmetry energy $L$ is shown as a function of the symmetry energy at saturation $S_{0}$. See text for details.

tory has been widely discussed [45]. The experiment should extract the value of the neutron radius in ${ }^{208} \mathrm{~Pb}$ from parityviolating electron scattering. However, the experimental signature is very small, and the extracted thickness has a large statistical uncertainty. In the next few years, a second experimental run for PREX could reduce this large uncertainty [46].

Recent experimental data obtained by Zenihiro et al.[47] on the neutron skin thickness of ${ }^{208} \mathrm{~Pb}$ deduced a value $\delta R_{n p}=$ $0.211_{-0.063}^{+0.054} \mathrm{fm}$. From the experiments constraints on the symmetry energy were derived, and these are plotted in Fig 3 as the short-dashed blue rectangular box labelled $\mathrm{Pb}(\vec{p}, \vec{p})$.

Last, we mention the experimental data on the Pygmy Dipole Resonance (PDR) in very neutron-rich nuclei such as ${ }^{68} \mathrm{Ni}$ and ${ }^{132} \mathrm{Sn}$, which peaks at excitation energies well below the Giant Dipole Resonance (GDR), and exhausts about $5 \%$ of the energy-weighted sum rule [48]. In many models it has been found that this percentage is linearly dependent on the slope $L$ of the symmetry energy. Carbone et al. [49] extracted a value of $L=64.8 \pm 15.7 \mathrm{MeV}$, and $S_{0}=32.2 \pm 1.3$ $\mathrm{MeV}$ using various models which connect $L$ with the neutron skin thickness. Those constraints are shown as a long-dashed rectangle in Fig 3 with the label PDR.

The predictions of the different EoS are also reported in Fig 3 as full symbols. They are distributed within a large region and they span a wide interval in the values of the parameter $L$. However, the various phenomenological data are at best marginally compatible, and it is difficult to put well definite constraints on the EoS. Tentatively, from these data one can restrict the possible values of the symmetry energy at saturation in a limited interval, approximately $30<S_{0}<35 \mathrm{MeV}$, where all the considered EoS are actually falling. 

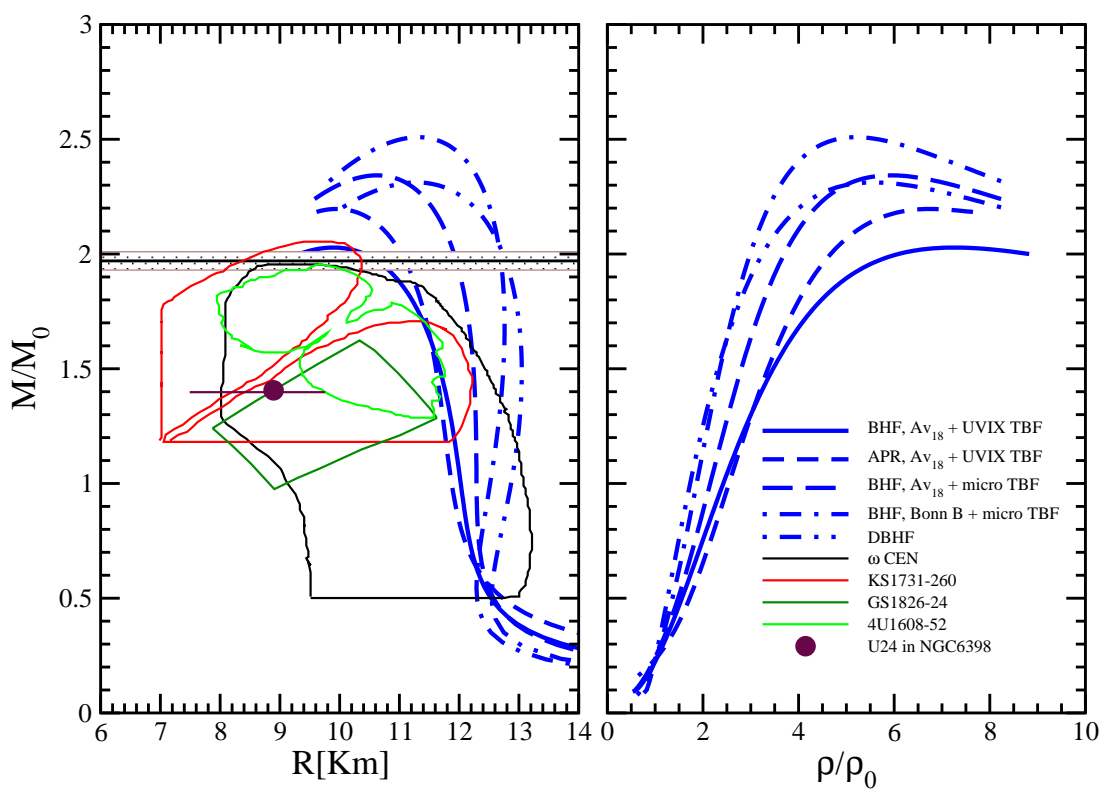

FIG. 4: (Color online) The mass-radius (left panel) and the mass-central density (right panel) relations are plotted for the EoS's discussed. Boxes are boundaries extracted from observations, see ref.[52].
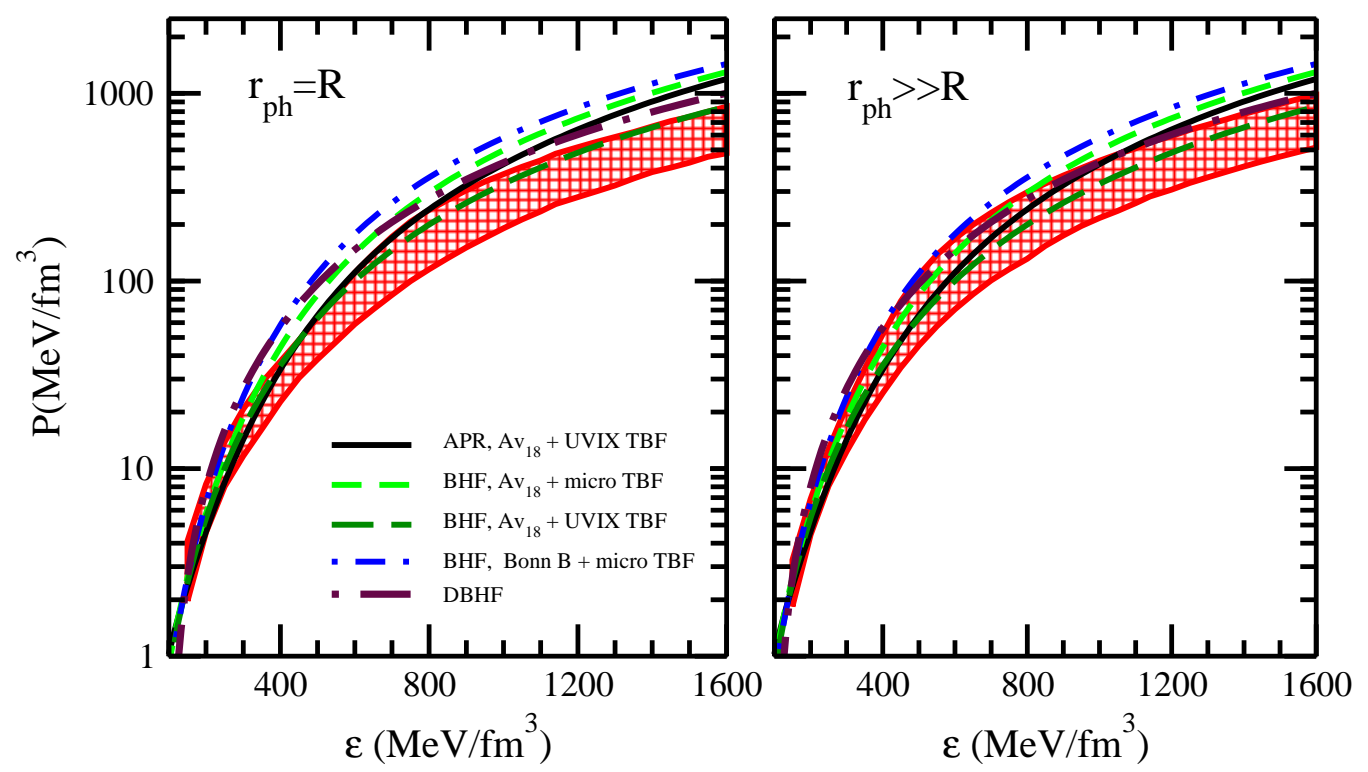

FIG. 5: (Color online) Pressure as a function of the mass-energy density in neutron star matter. The shaded areas are taken from ref.[54]. See text for details.

\section{Astrophysics}

A neutron star is bound by gravity, and it is kept in hydrostatic equilibrium only by the pressure produced by the compressed nuclear matter. It is then apparent that the nuclear matter EoS is the main medium property that is relevant in this case, as can be seen in the celebrated Tolman-Oppenheimer-
Volkoff [50] equations, valid for spherically symmetric NS

$$
\begin{aligned}
& \frac{d P}{d r}=-G \frac{\varepsilon m}{r^{2}}\left(1+\frac{P}{\varepsilon}\right)\left(1+\frac{4 \pi P r^{3}}{m}\right)\left(1-\frac{2 G m}{r}\right)^{-1} \\
& \frac{d m}{d r}=4 \pi r^{2} \varepsilon
\end{aligned}
$$

where $G$ is the gravitational constant, $P$ the pressure, $\varepsilon$ the energy density, and $r$ the (relativistic) radius coordinate. To close the equations we need the relation between pressure and density, $P=P(\varepsilon)$, i.e. just the EoS. Integrating these equations one gets the mass and radius of the star for each central 
density. Typical values are 1-2 solar masses $\left(M_{\odot}\right)$ and about $10 \mathrm{Km}$, respectively. This indicates the extremely high density of the object. It turns out that the mass of the NS has a maximum value as a function of radius (or central density), above which the star is unstable against collapse to a black hole. The value of the maximum mass depends on the nuclear EoS, so that the observation of a mass higher than the maximum one allowed by a given EoS simply rules out that EoS. The considered microscopic EoS's are compatible with the largest mass observed up to now, that is close to $1.97 \pm 0.04 M_{\odot}$ [51]. This is clearly shown in Fig 4 , where the mass-radius (left panel) and mass-central density relations (right panel) are plotted for all the considered EoS's as thick lines. It looks unlikely that this value is indeed the largest possible NS mass, and therefore future observational data on NS masses could overcome this limit and strongly constrain the nuclear EoS.

It would be of course desirable to have some phenomenological data also on the radius of NS. Unfortunately this is quite difficult, but some tentative analysis looks promising [52, 53]. In Fig 4 a sample of observational data taken from ref.[52] is displayed by closed thin lines for different sources, measured in quiescence and from thermonuclear bursts. It turns out that the current measurements are consistent with radii in the range $8-12 \mathrm{~km}$ and disfavor neutron stars with $\mathrm{R}$ $\sim 15 \mathrm{~km}$. Those measurements are consistent with the recent observation of the neutron star in SAX J1748.9-2021, which points to the neutron star radius in the $8-11 \mathrm{~km}$ range [53].

Additional tentative constraints on the nuclear EoS were obtained in a recent analysis of the data on six NS based on Bayesian statistical framework [54]. Depending on the hypothesis made on the structure of the NS, the results are slightly different, as shown in Fig[5] where the quantity $r_{p h}$ is the photosphere radius. In the let panel $r_{p h}$ is comparable to the neutron star radius $\mathrm{R}$, whereas in the right panel a substantial expansion of the photosphere during an X-ray burst is assumed to occur. The overall allowed region where the EoS's should lie is displayed in Fig 5 as bounding boxes, where the theoretical EoS's just discussed are also reported as thick lines. Among the different EoS's, only the one calculated with BHF and phenomenological Urbana model appear to be compatible with the extracted observational constraints over the whole density range. It turns out that other microscopic EoS do not show the same agreement, in particular the EoS with BHF and microscopic TBF and the DBHF EoS look too repulsive at high density. These boundaries obtained from astrophysical data are complementary to the ones obtained from heavy ion reactions, and illustrated in the previous subsection. In fact, in heavy ion collisions the tested matter is essentially symmetric, while in NS the matter is highly asymmetric. Considered together, the two types of constraints probe the density dependence of the symmetry energy.

In relation to the high density region of the nuclear EoS, an additional test is on the speed of sound $c_{S}$, that is required to be smaller than the speed of light $c$ (causality condition). The speed of sound is directly connected with the incompressibility and the energy density, according to the relativistic expres-

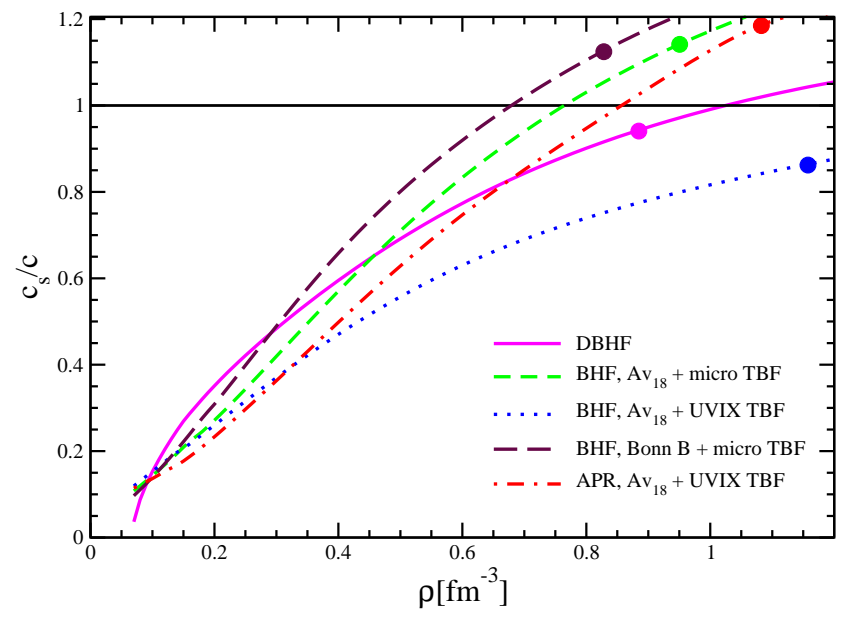

FIG. 6: (Color online) The speed of sound is plotted as function of the nucleon density for the EoS's discussed in the text. The dots mark the central density for the maximum mass of a neutron star.

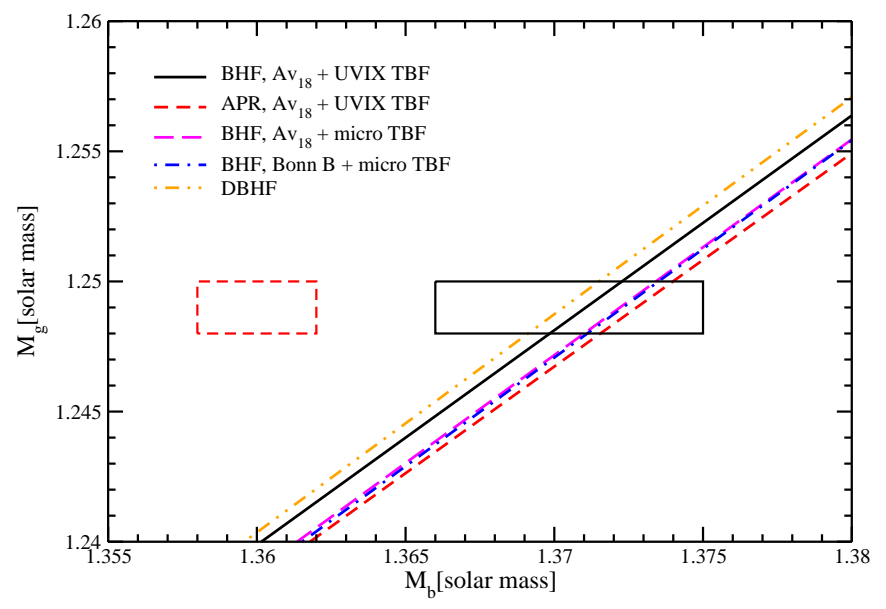

FIG. 7: (Color online) The gravitational mass is plotted as a function of the baryon mass for the EoS's discussed in the text. The boxes indicate the boundaries coming from the simulations.

sion

$$
\frac{c_{S}}{c}=\sqrt{\frac{d P}{d \varepsilon}}
$$

As such, it depends on the matter energy density and asymmetry. In Fig 6 we plot the speed of sound in units of $c$ as a function of the density of the NS matter, according to each EoS. In the figure the full points on each curve indicate the central density of the calculated NS maximum mass for the given EoS. At large enough density most of the EoS's show a superluminal speed of sound. One finds that also the DBHF EoS shows a superluminal behavior. This is not completely surprising, since the DBHF approach is actually based on the three-dimensional reduction of the original four-dimensional Bethe-Salpeter equation, and therefore it is not fully relativistic.

A further additional constraint on the neutron star EoS is provided by the observation of the double pulsar J0737-3039, 
and the interpretation given by Podsiadlowski [55]. In fact, the gravitational mass of Pulsar B is very precisely known $\mathrm{M}_{\mathrm{G}}=1.249 \pm 0.001 \mathrm{M}_{\odot}$, whereas estimates of the baryonic mass depend upon its detailed mode of formation. As modelled by Podsiadlowski et al., if the pulsar B was formed from a white dwarf with an $\mathrm{O}-\mathrm{Ne}-\mathrm{Mg}$ core in an electron-capture supernova, assuming no or negligible loss of baryonic mass during the collapse, the newly born neutron star will have the same baryonic mass as the precollapse core of the progenitor star, i.e. $\mathrm{M}_{\mathrm{B}} \simeq 1.366-1.375 \mathrm{M}_{\odot}$. This result is displayed in Fig 7 as a black box. Though, taking into account the uncertainty in the EoS and the small mass loss during the collapse, Kitaura et al. [56] made another simulation which gave $\mathrm{M}_{\mathrm{B}}=1.360 \pm 0.002 \mathrm{M}_{\odot}$, which is shown in Fig 7 by the dashed red box. We have calculated for each neutron star matter EoS the relation between the gravitational and baryonic mass, and these are displayed in Fig 7 by the straight curves. We notice that the results of all microscopic EoS's agree very well with the result of Podsiadlowski, at variance with the calculations based on the phenomenological Skyrme forces discussed in ref.[4], where agreement was found with the result of Kitaura et al. [56], which assumed small mass loss during the collapse.

\section{CONCLUSIONS}

We have presented a systematic confrontation of the nuclear Equation of State, obtained within different microscopic many-body methods, with the available constraints coming from phenomenology. The latter are extracted from laboratory experiments as well as from astrophysical observations. Both nuclear structure and heavy ion collisions data were considered, along the same lines of the analysis on the Skyrme forces reported in ref. [4]. Astrophysical observational data included the measures of NS masses, some hints on the radiusmass relation from ref. [52, 53] and the constraints on the EoS presented in ref. [54], obtained from the analysis of transient phenomena in six NS's. Some theoretical constraints, as the requirement of a sub-luminal speed of sound, were also considered. If one takes literally all the constraints, among the considered microscopic EoS only one passes all the tests. However, these phenomenological constraints are affected by uncertainties, which are difficult to estimate quantitatively on a firm basis. The conclusion that one can draw from this analysis is twofold. Firstly, despite the differences between the considered microscopic EoS, their overall predictions do not show major discrepancies with one another as well as with the phenomenological constraints. In other words, the calculated microscopic nuclear EoS, based on different many-body methods, are not in contradiction with the phenomenological constraints. This is not at all an obvious result. Secondly, from the analysis it appears that one can explain reasonably well all the data with a microscopic EoS that includes only nucleonic degrees of freedom, in particular no exotic components in NS are needed. On one hand, it is likely that this conclusion will be disproved in the future with the expected new data, in particular on the NS maximum mass. Indeed, if the evidence for a NS mass of 2.5 solar mass [57] will be confirmed, this will rule out most of the considered microscopic EoS and will introduce a serious and fundamental issue in the physics of NS. On the other hand, it has been shown that exotic matter like hyperons or quarks should appear in NS [22], which will strongly affect the EoS and challenge the theory of high density nuclear matter. In the future one can expect that the interplay between theory and observations will continue to play a major role in the worldwide effort of determining the nuclear EoS.

\section{Acknowledgments}

We warmly thank P. Danielewicz and J. Lee for providing us the data on the symmetry energy shown in Fig 2 Moreover we acknowledge H.-J. Schulze for the data concerning the EoS calculated in the BHF approach with Bonn B NN potential and microscopic TBF's, and C. Fuchs for the DBHF data set.
[1] J. Aichelin and J. Schaffner-Bielich, arXiv:0812.1341 Relativistic Heavy Ion Physics, Landolt-Börnstein - Group I Elementary Particles, Nuclei and Atoms, 23 (2010) 88, SpringerVerlag Berlin Heidelberg.

[2] Li Bao-An, Chen Lie-Wen and Ko Che Ming, Phys. Rep. 464, 113 (2008).

[3] M. Baldo, and G. F. Burgio, Rep. Prog. Phys. 75, 026301 (2012).

[4] M. Dutra, O. Laurenço, J. S. Sá Martins, A. Delfino, J. R. Stone and P. D. Stevenson, Phys. Rev. C 85, 035201 (2012).

[5] P. D. Stevenson, P. M. Goddard, J. R. Stone and M. Dutra, arXiv:1210.1592. Proceedings of XXXV Reunião de Trabalho sobre Física Nuclear no Brasil, 2012.

[6] T. Klähn, D. Blaschke, S. Typel, E. N. E. van Dalen, A. Faessler, C. Fuchs, T. Gaitanos, H. Grigorian, A. Ho, E. E. Kolomeitsev,
M. C. Miller, G. Röpke, J. Trümper, D. N. Voskresensky, F. Weber, and H.H. Wolter, Phys. Rev. C 74, 035802 (2006).

[7] M. Baldo, Nuclear Methods and the Nuclear Equation of State, International Review of Nuclear Physics, Vol. 8 (World Scientific, Singapore, 1999).

[8] R. Machleidt, Advances in Nuclear Physics 19, 189 (1989).

[9] V. R. Pandharipande and R. B. Wiringa, Rev. Mod. Phys. A 51, 821 (1979).

[10] J.A. Oller, Sixth International Conference on Quarks and $\mathrm{Nu}-$ clear Physics, Proceedings of Science 2012, arXiv:1206.2449

[11] A.Lacour, U.-G. Meissner and J. A. Oller, AIP Conf.Proc.1322:125-133 2010, arXiv:1009.2957

[12] A. Lacour, J. A. Oller, U.-G. Meissner, Annals of Physics 326, 241 (2011).

[13] K. Heeler, S. K. Bogner, R. J. Furnsthal, A. Nogga and A. 
Schwenk, Phys. Rev. C 83, 031301 (2011).

[14] N. Kaiser, S. Fritsch and W. Weise, Nucl. Phys. A697, 255 (2002).

[15] S. Fritsch, N. Kaiser and W. Weise, Nucl. Phys. A750, 259 (2005).

[16] R. Machleidt, P. Liu, D. R. Entem and E. Ruiz Arriola, Phys. Rev. C81, 024001 (2010).

[17] H. Q. Song, M. Baldo, G. Giansiracusa, and U. Lombardo, Phys. Rev. Lett. 81, 1584 (1998); M. Baldo, G. Giansiracusa, U. Lombardo, and H. Q. Song, Phys. Lett. B473, 1 (2000); M. Baldo, A. Fiasconaro, H. Q. Song, G. Giansiracusa, and U. Lombardo, Phys. Rev. C65, 017303 (2001).

[18] M. Baldo, I. Bombaci, and G. F. Burgio, Astron. Astrophys. 328, 274 (1997).

[19] J. Carlson, V. R. Pandharipande, and R. B. Wiringa, Nucl. Phys. A401, 59 (1983); R. Schiavilla, V. R. Pandharipande, and R. B. Wiringa, Nucl. Phys. A449, 219 (1986); B. S. Pudliner, V. R. Pandharipande, J. Carlson, S. C. Pieper, and R. B. Wiringa, Phys. Rev. C56, 1720 (1997).

[20] Z. H. Li and H.-J. Schulze, Phys. Rev. C78, 028801 (2008).

[21] P. Grangé, A. Lejeune, M. Martzolff, and J.-F. Mathiot, Phys. Rev. C40, 1040 (1989); W. Zuo, A. Lejeune, U. Lombardo, and J.-F. Mathiot, Nucl. Phys. A706, 418 (2002); Z. H. Li, U. Lombardo, H.-J. Schulze, and W. Zuo, Phys. Rev. C77, 034316 $(2008$

[22] H.-J. Schulze, M. Baldo, U. Lombardo, J. Cugnon, and A. Lejeune, Phys. Rev. C57, 704 (1998); M. Baldo, G. F. Burgio, and H.-J. Schulze, Phys. Rev. C58, 3688 (1998); Phys. Rev. C61, 055801 (2000).

[23] G. E. Brown, W. Weise, G. Baym and J. Speth, Commun. Nucl. Part. Phys. 17, 39 (1987).

[24] Z. H. Li, U. Lombardo, H.-J. Schulze, W. Zuo, L. W. Chen and H. R. Ma, Phys. Rev. C 74 (2006), 047304.

[25] T. Gross-Boelting, C. Fuchs, and A. Faessler, Nucl. Phys. A 648, 105 (1999).

[26] J. Navarro, R. Guardiola and I. Moliner, Introduction to Modern Methods of Quantum Many-Body Theory and their Applications (World Scientific, Singapore, 2002).

[27] A. Akmal, V. R. Pandharipande, and D. G. Ravenhall, Phys. Rev. C58, 1804 (1998).

[28] R. B. Wiringa, V. G. J. Stoks, and R. Schiavilla, Phys. Rev. C51, 38 (1995).

[29] M. Baldo, L. Robledo, P. Schuck and X. Vinas, arXiv:1210.1321.

[30] A.W. Steiner, M. Prakash, J.M. Lattimer and P.J. Ellis, Phys. Rep. 411, 325 (2005).

[31] H. Heiselberg, and M. Hjorth-Jensen, Phys. Rep. 328, 237 (1999).

[32] J. P. Blaizot, Phys. Rep. 64, 171 (1980).

[33] D. Miskowiec et al., Phys. Rev. Lett. 72, 3650 (1994).

[34] J. Ritman et al., Z. Phys. A352, 355 (1995).

[35] C. Fuchs, Prog. Part. Nucl. Phys. 56, 1 (2006).

[36] P. Danielewicz, R. Lacey and W. Lynch, Science 298, 1592 (2002).

[37] M. B. Tsang, J. R. Stone, F. Camera, P. Danielewicz, S. Gandolfi, K. Hebeler, C. J. Horowitz, Jenny Lee, W. G. Lynch, Z. Kohley, R. Lemmon, P. Möller, T. Marukami, S. Riordan, X. Roca-Maza, F. Sammarruca, A. W. Steiner, I. Vidana, and S. J. Yennello, Phys. Rev. C86, 015803 (2012)

[38] P. Danielewicz and J. Lee, private communication.

[39] M. Famiano, T. Liu, W. G. Lynch, M. Mocko, A. M. Rogers,
M. B. Tsang, M. S. Wallace, R. J. Charity, S. Komarov, D. G. Sarantites, L. G. Sobotka, and G. Verde, Phys. Rev. Lett. 97, 052701 (2006).

[40] M. B. Tsang, C. K. Gelbke, X. D. Liu, W. G. Lynch, W. P. Tan, G. Verde, H. S. Xu, W. A. Friedman, R. Donangelo, S. R. Souza, C. B. Das, S. D. Gupta, and D. Zhabinsky, Phys. Rev. C 64, 054615 (2001).

[41] T. X. Liu, W. G. Lynch, M. B. Tsang, X. D. Liu, R. Shomin, W. P. Tan, G. Verde, A. Wagner, H. F. Xi, H. S. Xu, B. Davin, Y. Larochelle, R. T. de Souza, R. J. Charity, and L. G. Sobotka, Phys. Rev. C 76, 034603 (2007).

[42] Z. Y. Sun, M. B. Tsang, W. G. Lynch, G. Verde, F. Amorini, L. Andronenko, M. Andronenko, G. Cardella, M. Chatterje, P. Danielewicz, E. De Filippo, P. Dinh, E. Galichet, E. Geraci, H. Hua, E. La Guidara, G. Lanzalone, H. Liu, F. Lu, S. Lukyanov, C. Maiolino, A. Pagano, S. Piantelli, M. Papa, S. Pirrone, G. Politi, F. Porto, F. Rizzo, P. Russotto, D. Santonocito, and Y. X. Zhang, Phys. Rev. C 82, 051603(R) (2010).

[43] Z. Kohley, L. W. May, S. Wuenschel, M. Colonna, M. Di Toro, M. Zielinska-Pfabe, K. Hagel, R. Tripathi, A. Bonasera, G. A. Souliotis, D. V. Shetty, S. Galanopoulos, M. Mehlman, W. B. Smith, S. N. Soisson, B. C. Stein, and S. J. Yennello, Phys. Rev. C 83, 044601 (2011).

[44] P. Möller, W. D. Meyers, H. Sagawa, and S. Yoshida, Phys. Rev. Lett. 108, 052501 (2012).

[45] C. J. Horowitz, Phys. Rev. C 57, 3430 (1998); Eur. Phys. J. A 30, 303 (2006); C. Horowitz, S. J. Pollock, P. A. Souder, and R. Michaels, Phys. Rev. C 63, 025501 (2001).

[46] P. A. Souder, R. Holmes, Chun-Min Jen, L. Zana, Z. Ahmed, A. Rakhman, E. Cisbani, S. Frullani, F. Garibaldi, F. Meddi et al., PREX II experimental proposal to Jefferson Laboratory, PAC38, http://hallaweb.jlab.org/parity/prex/prexII.pdf

[47] J. Zenihiro, H. Sakaguchi, T. Murakami, M. Yosoi, Y. Yasuda, S. Terashima, Y. Iwao, H. Takeda, M. Itoh, H. P. Yoshida, and M. Uchida, Phys. Rev. C 82, 044611 (2010); J. Zenihiro, Ph.D. Thesis, Kyoto University, 2011.

[48] A. Klimkiewicz, N. Paar, P. Adrich, M. Fallot, K. Boretzky, T. Aumann, D. Cortina-Gil, U. Datta Pramanik, Th. W. Elze, H. Emling, H. Geissel, M. Hellstro?m, K. L. Jones, J. V. Kratz, R. Kulessa, C. Nociforo, R. Palit, H. Simon, G. Suro?wka, K. Su?mmerer, D. Vretenar, and W. Walus?, Phys. Rev. C 76, 051603(R) (2007).

[49] A. Carbone, G. Colo', A. Bracco, L.-G. Cao, P. F. Bortignon, F. Camera, and O. Wieland, Phys. Rev. C 81, 041301 (2010).

[50] S. L. Shapiro and S. A. Teukolsky, Black Holes, White Dwarfs, and Neutron Stars (John Wiley \& Sons, New York, 1983).

[51] P. B. Demorest, T. Pennucci, S. M. Ransom, M. S. E. Roberts, and J. W. T. Hessels, Nature 467, 1081 (2010).

[52] F. Özel, Rep. Prog. Phys. 76, 016901 (2013).

[53] T. Guver, and F. Özel, Astrophys. J. 765, L1 (2013).

[54] A. W. Steiner, J. M. Lattimer, E. F. Brown, Astrophys. J. 722, 33 (2010).

[55] Ph. Podsiadlowski, J. D. M. Dewi, P. Lesaffre, J. C. Miller, W. G. Newton, and J. R. Stone, Mon. Not. R. Astron. Soc. 361, 1243 (2005).

[56] F. S. Kitaura, H.-Th. Janka, and W. Hillebrandt, Astron. Astrophys. 450, 345 (2006).

[57] R. W. Romani, A. V. Filippenko, J. M. Silverman, S. B. Cenko, J. Greiner, A. Rau, J. Elliott, and H. J. Pletsch, arXiv:1210.6884 